\title{
Does Intravesical Prostatic Protrusion Affect Oncological Outcomes and Toxicity in Prostate Cancer Patients Receiving Definitive Radiotherapy?
}

\author{
Pervin HURMUZ ${ }^{1}$, Caglayan Selenge Beduk ESEN ${ }^{1}$, Emre UNAL ${ }^{2}$, \\ Musturay KARCAALTINCABA ${ }^{2}$, Gokhan OZYIGIT ${ }^{1}$, Husnu Fadil AKYOL ${ }^{1}$ \\ ${ }^{1}$ Hacettepe University, Faculty of Medicine, Department of Radiation Oncology \\ ${ }^{2}$ Hacettepe University, Faculty of Medicine, Department of Radiology, Ankara, TURKEY
}

\begin{abstract}
Intravesical prostatic protrusion (IPP) is associated with increased urinary toxicities after radical prostatectomy. The aim of the current study is to investigate the effect of IPP on treatment outcomes and toxicity in prostate cancer patients who underwent definitive radiotherapy (RT). Medical records of 130 patients who received RT between April 2007 and October 2017 were retrospectively evaluated. All patients received conventionally-fractionated intensity-modulated RT to a total dose of 70-78Gy. We found a very strong positive correlation with MRI and CT thus IPP grades for the whole cohort were evaluated using CT scans. Acute toxicities were evaluated using CTCAE version4.0 and late toxicities were evaluated using RTOG/EORTC guidelines. Mann-Whitney $U$, chi-square and student- $t$ tests were used for statistical analyses in SPSS version15.0. Forty-two patients did not have IPP, 19 patients had grade I, 47 patients had grade II and 22 patients had grade III IPP. There was no difference in age, PSA level and GS but prostate volume was higher in the IPP group ( $p=0.013$ ). With a median follow-up of 53.4 months, biochemical recurrences were observed in 10 patients in the IPP group and 2 patients in the non-IPP group $(p=0.334)$. There was no significant difference in treatment outcomes. RT was well tolerated however grade $\geq 2$ acute genitourinary $(G U)$ toxicity was higher in the IPP group $(p=0.024)$. CT scan is strongly correlated with MRI in terms of grading IPP. IPP does not affect RT outcomes however it seems to be a risk factor for acute GU toxicity.
\end{abstract}

Keywords: Intravesical prostatic protrusion, Radiotherapy, Prostate cancer

\section{INTRODUCTION}

Prostate cancer $(\mathrm{PCa})$ is the most common type of cancer among men with an estimated 174,650 new cases in 2019. ${ }^{1}$ External beam radiation therapy (EBRT) with or without androgen deprivation therapy (ADT) is one of the treatment options for clinically localized PCa. ${ }^{2,3}$ Although, new radiotherapy (RT) techniques like intensity-modulated radiotherapy (IMRT) and image-guided radiotherapy (IGRT) reduced gastrointestinal (GI) toxicities, significant reductions of urinary-related toxicities after high-dose RT have not been observed yet. ${ }^{4,5}$ Frequency of $\geq$ grade 2 acute and late genitourinary $(\mathrm{GU})$ related toxicities (including erectile impotence, urinary frequency/urgency and urinary incontinence) in PCa patients who underwent definitive IMRT is approximately $11 \%$ and $69 \%$ respectively. ${ }^{5}$ In the literature, a few studies reported increased radiation dose to the lower bladder and bladder trigone seemed to be associated with increased GU toxicity. ${ }^{6,7}$ Previously, the presence of diabetes mellitus, receiving anticoagulant treatment, increased RT dose were found to be associated with increased late GU toxicity. ${ }^{8}$ 


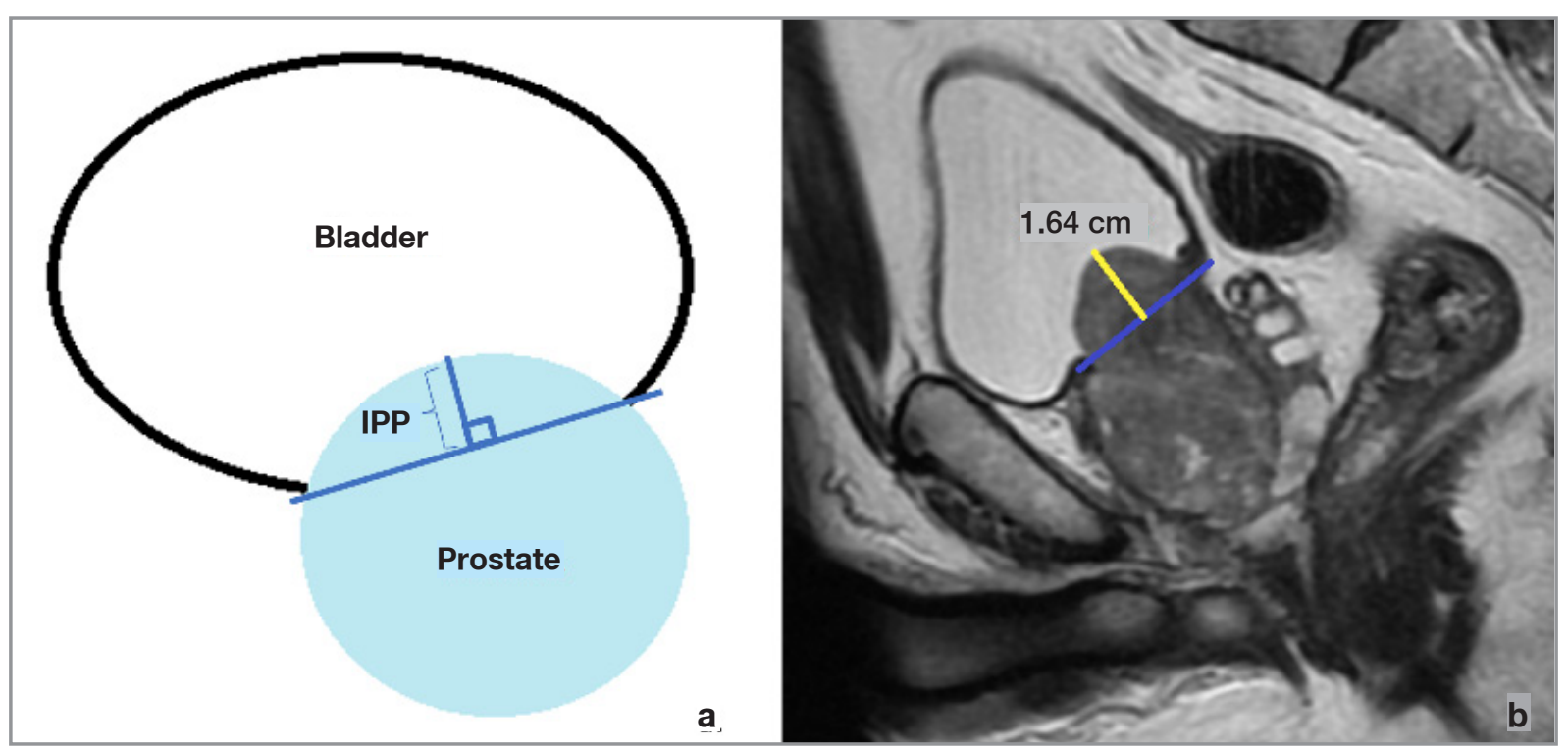

Figure 1. Measurement of the intravesical prostatic protrusion (IPP). (a) Schematic estimation of IPP: The vertical distance from the tip of the protruding prostate to the base of the bladder. (b) Sagittal view of magnetic resonance imaging (MRI) and protruding prostate

Intravesical prostatic protrusion (IPP) was found to be correlated with bladder outlet obstruction and decreased International Prostate Symptom Score (IPSS) after transurethral resection of the prostate (TURP) for benign prostatic hyperplasia. ${ }^{9,10}$ Although IPP was previously found to be associated with increased urinary incontinence rates and prolonged duration of postoperative urinary incontinence after radical prostatectomy ${ }^{11}$, there is no evidence that IPP affects oncological outcomes and early or late GU toxicity in patients who underwent definitive radiotherapy. Previously, IPP was evaluated by using ultrasonography or magnetic resonance imaging (MRI), there is no evidence that IPP can be evaluated by computed tomography (CT). Therefore, the present study aimed to evaluate the correlation of IPP grades between MRI and CT scans and the effect of IPP on oncological outcomes and early or late toxicity after radiotherapy.

\section{PATIENTS AND METHODS}

Medical records of 130 patients who underwent definitive RT for localized PCa between April 2007 and October 2017 were retrospectively evaluated. All patients received conventionally fractionated IMRT to a total dose of 70-78 Gy using the Brainlab® Novalis system. Radiotherapy planning CT scans were obtained when the bladder was filled with $500 \mathrm{ml}$ of saline solution. All of the evaluations were made using planning CT. Since the patients with lymph node involvement were not included in the present study, lymphatic irradiation was not performed in any of the patients. The clinical target volume (CTV) was defined as the whole prostate gland with or without bilateral SV. The extent of the delineation of SVs depended on the D'Amico risk group stratification system. ${ }^{12}$ In intermediate-risk disease, proximal SVs were countered, whereas in high-risk disease whole SVs were countered. CTV was enlarged with a $0.5 \mathrm{~cm}$ margin in anterior, left and right directions and 0.3 $\mathrm{cm}$ in the posterior direction to constitute planning target volume (PTV). Bladder volume that receives 70 Gy (V70) and $40 \mathrm{~Gy}$ (V40) was allowed to be < $35 \%$ and $<40 \%$ of all bladder volume and rectum volume that receives 65 Gy (V65) and 40 Gy (V40) was allowed to be $35 \%$ and $40 \%$ of all rectum volume in treatment planning. ${ }^{13}$

IPP was retrospectively evaluated by two experienced radiologists using diagnostic multiparametric MRI and CT scans. Since not all patients had diagnostic multiparametric MRI, firstly the correlation of IPP between MRI and CT images were evaluated in 49 patients who had both MRI and CT scans. According to Gravas et al IPP was defined as the vertical distance from the tip of the protrusion 


\begin{tabular}{|c|c|c|c|c|c|}
\hline & & \multicolumn{3}{|c|}{ MRI } & \multirow[b]{2}{*}{ Grade III IPP (n) } \\
\hline & & No IPP (n) & Grade I IPP (n) & Grade II IPP (n) & \\
\hline \multirow[t]{4}{*}{ CT } & No IPP (n) & 12 & 5 & 5 & 0 \\
\hline & Grade I IPP (n) & 1 & 2 & 5 & 0 \\
\hline & Grade II IPP (n) & 0 & 2 & 8 & 1 \\
\hline & Grade III IPP (n) & 0 & 0 & 1 & 7 \\
\hline
\end{tabular}

to the base of the bladder in the sagittal plane and graded as grade I if the distance was $<5 \mathrm{~mm}$, grade II if the distance was 5-10 $\mathrm{mm}$ and grade III if the distance was $>10 \mathrm{~mm}$ (Figure 1). ${ }^{14}$

We divided 130 patients into two groups according to the presence of IPP in CT scans. Acute side effects were evaluated using CTCAE version 4.0 and late side effects were evaluated using RTOG/ EORTC guidelines. Patients had a PSA test every 3 months in the first 2 years following RT and every 6 months thereafter. Biochemical recurrence was defined as PSA nadir $+2 \mathrm{ng} / \mathrm{mL}$ based on the Phoenix definition. ${ }^{15}$

Statistical analysis was performed with SPSS version 20 (SPSS Inc., Chicago, IL, USA) and statistical significance was defined as a $\mathrm{p}$ value of $<0.05$. The variables were investigated using visual (histograms, probability plots) and analytical methods (Kolmogorov-Simirnov/Shapiro-Wilk's test) to determine whether or not they are normally distributed. While investigating the associations between non-normally distributed and/or ordinal variables, the correlation coefficients and their significance were calculated using the Spearman test. The Mann-Whitney U test was used to compare nonnormally distributed and ordinal variables between the groups. Student's t-test was used to compare normally distributed variables between the groups. This retrospective study was conducted in compliance with the principles of Helsinki declaration and informed consent was obtained from each patient.

Ethical approval for this retrospective study was obtained from the Institutional Review Board of Hacettepe University (IRB Decision number: 2020/04-24).

\section{RESULTS}

The median age of the patients was 71.5 years (range $=45-86$ years), median GS was 7.5 (range $=$ 6-10), median PSA level at diagnosis was $28 \mathrm{ng} / \mathrm{mL}$ (range $=2-374 \mathrm{ng} / \mathrm{mL}$ ) and median prostate volume was $46.25 \mathrm{~mL}$ (range $=11-166 \mathrm{~mL}$ ). There were 10 patients $(7.7 \%)$ in the low-risk, 22 patients $(16.9 \%)$ in the intermediate-risk and 98 patients (75.4\%) in the high-risk group according to D'Amico risk group stratification system. After RT, 118 patients received $\mathrm{ADT}$ of median 12 months (range $=3-84$ months). Since not all patients had diagnostic multiparametric MRI, we first evaluated the correlation of IPP between pretreatment MRI and CT images in 49 patients that have both MRI and CT scans. MRI and CT showed a very strong positive correlation for IPP grades $(r=0.758, p<0.001)$ and the IPP grades were given in detail for both MRI and CT scans in Table 1.

Thus IPP grades for the whole cohort were evaluated in CT scans by the same radiologists; 42 patients (32.3\%) did not have IPP, 19 patients (14.6\%) had grade I, 47 patients $(36.2 \%)$ had grade II and 22 patients $(16.9 \%)$ had grade III IPP. There was no significant difference in age, PSA level, GS, duration of hormonal therapy and the volume of SVs in RT field between IPP and non-IPP groups but prostate volume was found significantly higher in the IPP group ( $\mathrm{p}=0.013)$ (Table 2).

Median follow-up time was 53.4 months (8.5148.9 months). Median survival was not achieved during the follow-up period. Biochemical recurrences (BCR) were observed in 10 patients $(11.9 \%)$ in the IPP group and 2 patients (4.8\%) in the nonIPP group ( $\mathrm{p}=0.334)$. There was no significant dif- 
International Journal of Hematology and Oncology

Table 2. Comparison of patient characteristics between IPP and non-IPP groups

\begin{tabular}{|llll}
\hline Median (IQR) & IPP group $(\mathbf{n = 4 2 )}$ & Non-IPP group $(\mathbf{n = 8 8})$ & P value \\
\hline Age & $72(66-76)$ & $70(66-75)$ & 0.275 \\
PSA level (ng/ml) & $31(18-98)$ & $24.5(12.25-79.75)$ & 0.464 \\
Gleason Score & $8(7-9)$ & $7(7-9)$ & 0.730 \\
Duration of hormonal therapy (months) & $12(10-24)$ & $12(9-24)$ & 0.656 \\
Prostate volume $(\mathrm{mL})$ & $48.65(34.55-63.93)$ & $34.25(22.30-56.65)$ & $0.013^{\star}$
\end{tabular}

* Mann-Withney U test; statistical significance was defined as $p<0.005$

ference in 5-year overall survival (OS) $(78.4 \%$ vs $91 \% ; \mathrm{p}=0.559)$ and biochemical recurrence-free survival (bRFS) rates $(83.3 \%$ vs $90.6 \%$; $=0.183$ ) between IPP group and non-IPP group (Figure 2).

The treatment was well tolerated and no acute or late grade 3-4 toxicity was observed in this study. Frequency in 24 patients, dysuria in 31 patients, transient urinary incontinence in 3 patients and urgency in 1 patient were observed as acute GU toxicities. Proctitis was observed in 5 patients as acute GI toxicity. Urinary incontinence was observed in 2 patients as both acute and late GU toxicity. Dysuria in 3 patients, frequency in 16 patients, erectile dysfunction in 2 patients, hematuria in 1 patient, urinary incontinence in 5 patients and urgency in 2 patients were observed as late GU toxicities. Acute GI toxicity, late GI toxicity, and late GU toxicity rates were similar between the IPP and non-IPP groups. However grade $\geq 2$ acute GU toxicity was significantly higher in the IPP group than the nonIPP group $(\mathrm{p}=0.024)$. For the whole cohort median V70 and V40 for bladder was 7\% (0-33\%) and $28 \%(4 \%-63 \%)$ respectively. Median V65 and V40 for rectum was $9 \%(2 \%-24 \%)$ and $33 \%$ (13\%-55\%) respectively. No significant difference was observed in V70 or V40 for bladder ( $\mathrm{p}=0.137$, $\mathrm{p}=0.267$ respectively) and $\mathrm{V} 65$ or $\mathrm{V} 40$ for rectum $(\mathrm{p}=0.090, \mathrm{p}=0.088$ respectively) between IPP and non-IPP groups.

\section{DISCUSSION}

IPP occurs as the prostate gland enlarges into the bladder with median lobe hypertrophy. Ultrasonography and MRI are useful and non-invasive methods for measurement of IPP. ${ }^{10,16}$ In this study, since not all patients had MRI, the correlation of IPP grades between pretreatment diagnostic MRI and planning CT was performed initially and CT was found to be well correlated with MRI in terms of evaluating IPP grades. Subsequently IPP grades of all patients were evaluated using pre-treatment CT scans and it was found that IPP was associated with higher acute grade $\geq 2$ GU toxicity without interfering oncological outcomes.

Acute or late GU toxicity is one of the most common side effects after definitive RT in PCa patients that affects the quality of life. ${ }^{5}$ Presence of diabetes mellitus, receiving anticoagulant treatment, hypofractionation, older age, hormonal therapy, concurrent chemotherapy, increased RT dose to the lower bladder and bladder trigone was previously found to be associated with GU toxicity after definitive RT. ${ }^{6,8,17-19}$ The current study showed that IPP was associated with high rates of grade $\geq 2$ acute GU toxicity in PCa patients who underwent definitive RT. The patients' characteristics such as age, duration of hormonal therapy were similar between groups. None of the patients had hypo-fractionated RT or concurrent chemotherapy in the current study. The difference in GU toxicities seems to be caused by the presence of IPP. Although prostate volume is higher in the IPP group that might affect the volume of bladder in the RT field we couldn't find a difference in bladder dose-volume parameters in two groups. However there might be currently unknown different parameters that should be used to estimate the difference that leads to acute GU toxicities. To the best of our knowledge, this is the first evidence about the association between the presence of IPP and GU toxicity in PCa patients who underwent definitive RT. 


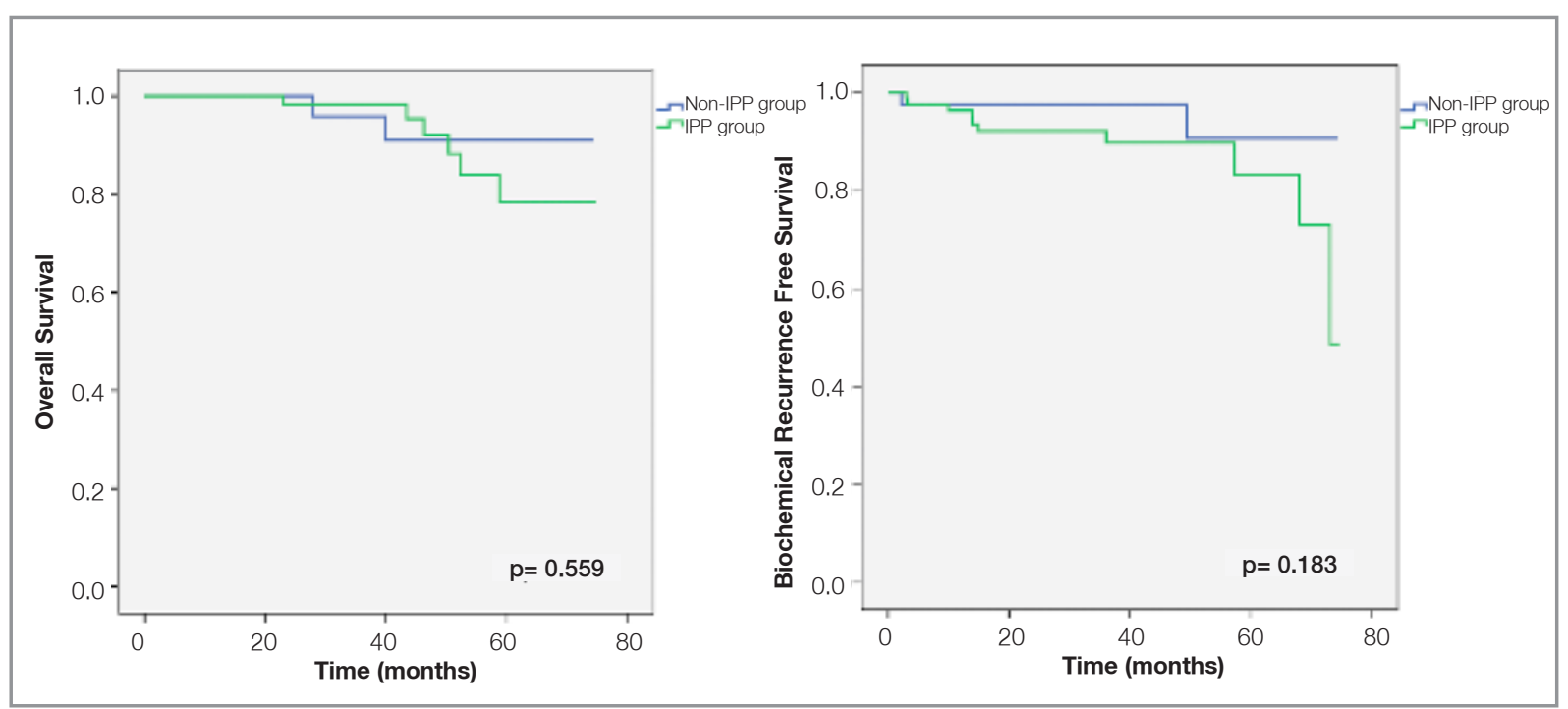

Figure 2. Comparison of 5-year overall survival and biochemical recurrence-free survival between the IPP group and non-IPP group.

Although a few studies are supporting that IPP was associated with bladder outlet obstruction in benign prostatic hyperplasia, there are controversial reports about the association of IPP and recovery of urinary incontinence after robot-assisted radical prostatectomy. ${ }^{20-22}$ Lee et al. showed that although IPP was associated with higher rates of urinary incontinence after laparoscopic radical prostatectomy, it was also found as a predictor of early urinary continence recovery. ${ }^{11}$ Jo et al, also reported that the presence and grade of IPP were related to low postoperative continence rates after robot-assisted laparoscopic radical prostatectomy. ${ }^{22}$ Hamidi et al. reported the presence of IPP did not affect oncological outcomes but was a disadvantage in gaining early urinary continence after robot-assisted radical prostatectomy. ${ }^{21}$

The current study showed no difference in 5-year OS and bRFS between the groups that are similar to literature for patients treated with surgery. However it should be kept in mind that IPP might be correlated with positive surgical margins at the base during robot-assisted laparoscopic radical prostatectomy. ${ }^{21,23}$

To the best of our knowledge current study is the first study to find a correlation of MRI and CT in the evaluation of IPP grades. Strong correlation in between helps to use planning CT in IPP grading in the routine practice. Additionally the effect of IPP on GU toxicity after surgery has been reported before however this is the first study to show the association of IPP and GU toxicity after definitive $\mathrm{RT}$ in the IMRT era.

The current study has some limitations. Firstly, the data was collected retrospectively. The information about predicting factors for GU toxicity such as the presence of diabetes mellitus, receiving anticoagulant treatment could not be reached from the patient records. The second limitation of this study is evaluating urinary symptoms without using objective methods such as IPSS or the number of pads per day. Patient-reported medical records were used for evaluation of urinary incontinence status.

In conclusion $\mathrm{CT}$ scan was found to be strongly correlated with MRI in terms of grading IPP. In future studies, planning CT scans can be used for the measurement of IPP in PCa patients. The presence of IPP does not affect the treatment outcomes however it seems to be a risk factor for GU toxicity. Thus reduction in the prostate volume before radiotherapy might be a good option to avoid acute GU toxicity. 
International Journal of Hematology and Oncology

\section{Acknowledgement:}

This study was presented as an oral presentation at 6th Mediterranean Multidisciplinary Oncology Forum (MMOF) Congress" and "3rd International Congress on Oncological Sciences” (27 November- 1 December 2019, Antalya-Turkey)

\section{REFERENCES}

1. Siegel RL, Miller KD, Jemal A. Cancer statistics, 2019. CA Cancer J Clin 69: 7-34, 2019.

2. Bolla M, Van Tienhoven G, Warde $\mathrm{P}$, et al. External irradiation with or without long-term androgen suppression for prostate cancer with high metastatic risk: 10-year results of an EORTC randomised study. Lancet Oncol 11: 1066-1073, 2010.

3. Warde P, Mason M, Ding K, et al. Combined androgen deprivation therapy and radiation therapy for locally advanced prostate cancer: a randomised, phase 3 trial. Lancet 378: 2104-2111, 2011.

4. Rosewall T, Catton C, Currie G, et al. The relationship between external beam radiotherapy dose and chronic urinary dysfunction--a methodological critique. Radiother Oncol 97: 40-47, 2010.

5. Michalski JM, Yan Y, Watkins-Bruner D, et al. Preliminary toxicity analysis of 3-dimensional conformal radiation therapy versus intensity modulated radiation therapy on the highdose arm of the Radiation Therapy Oncology Group 0126 prostate cancer trial. Int J Radiat Oncol Biol Phys 87: 932938, 2013.

6. Ghadjar P, Zelefsky MJ, Spratt DE, et al. Impact of dose to the bladder trigone on long-term urinary function after highdose intensity modulated radiation therapy for localized prostate cancer. Int J Radiat Oncol Biol Phys 88: 339-344, 2014.

7. Inokuchi H, Mizowaki T, Norihisa Y, et al. Correlation between urinary dose and delayed radiation cystitis after 78 Gy intensity-modulated radiotherapy for high-risk prostate cancer: A 10-year follow-up study of genitourinary toxicity in clinical practice. Clin Transl Radiat Oncol 6: 31-36, 2017.

8. Mathieu R, Arango JD, Beckendorf V, et al. Nomograms to predict late urinary toxicity after prostate cancer radiotherapy. World J Urol 32: 743-751, 2014.

9. Lee JW, Ryu JH, Yoo TK, et al. Relationship between Intravesical Prostatic Protrusion and Postoperative Outcomes in Patients with Benign Prostatic Hyperplasia. Korean J Urol 53: 478-482, 2012.

10. Nose H, Foo KT, Lim KB, et al. Accuracy of two noninvasive methods of diagnosing bladder outlet obstruction using ultrasonography: intravesical prostatic protrusion and velocityflow video urodynamics. Urology 65: 493-497, 2005.
11. Lee $\mathrm{CH}, \mathrm{Ha} \mathrm{HK}$. Intravesical prostatic protrusion as a predictor of early urinary continence recovery after laparoscopic radical prostatectomy. Int J Urol 21: 653-656, 2014.

12. D'Amico AV, Whittington R, Malkowicz SB, et al. Biochemical outcome after radical prostatectomy, external beam radiation therapy, or interstitial radiation therapy for clinically localized prostate cancer. JAMA 280: 969-974, 1998.

13. Michalski JM, Moughan J, Purdy J, et al. Effect of standard vs dose-escalated radiation therapy for patients with intermediate-risk prostate cancer: The NRG Oncology RTOG 0126 Randomized Clinical Trial. JAMA Oncol 4: e180039, 2018.

14. Gravas S, Cornu JNC, Gacci M, et al. Management of nonneurogenic male lower urinary tract symptoms (LUTS), incl. benign prostatic obstruction (BPO). European Association of Urology Guidelines. 2019.

15. Roach M 3rd, Hanks G, Thames H Jr. et al. Defining biochemical failure following radiotherapy with or without hormonal therapy in men with clinically localized prostate cancer: recommendations of the RTOG-ASTRO Phoenix Consensus Conference. Int J Radiat Oncol Biol Phys 65: 965-974, 2006.

16. Guneyli S, Ward E, Peng Y, et al. MRI evaluation of benign prostatic hyperplasia: Correlation with international prostate symptom score. J Magn Reson Imaging 45: 917-925, 2017.

17. Peeters ST, Hoogeman MS, Heemsbergen WD, et al. Volume and hormonal effects for acute side effects of rectum and bladder during conformal radiotherapy for prostate cancer. Int J Radiat Oncol Biol Phys 63: 1142-1152, 2005.

18. Levine LA, Richie JP. Urological complications of cyclophosphamide. J Urol 141: 1063-1069, 1989.

19. Moonen L, van der Voet $H$, Horenblas S, Bartelink H. A feasibility study of accelerated fractionation in radiotherapy of carcinoma of the urinary bladder. Int J Radiat Oncol Biol Phys 37: 537-542, 1997.

20. Chia SJ, Heng CT, Chan SP, Foo KT. Correlation of intravesical prostatic protrusion with bladder outlet obstruction. BJU Int 91: 371-374, 2003.

21. Hamidi N, Atmaca AF, Canda AE, et al. Does Presence of a Median Lobe Affect Perioperative Complications, Oncological Outcomes and Urinary Continence Following Robotic-assisted Radical Prostatectomy? Urol J 15: 248-255, 2018.

22. Jo JK, Hong SK, Byun SS, et al. Urinary Continence after Robot-Assisted Laparoscopic Radical Prostatectomy: The Impact of Intravesical Prostatic Protrusion. Yonsei Med J 57: 1145-1151, 2016.

23. Jeong CW, Lee S, Oh JJ, et al. Quantification of median lobe protrusion and its impact on the base surgical margin status during robot-assisted laparoscopic prostatectomy. World $\mathrm{J}$ Urol 32: 419-23, 2014. 


\section{Correspondence:}

\section{Dr. Pervin HURMUZ}

Hacettepe Universitesi, Tip Fakultesi

Radyasyon Onkolojisi Anabilim Dali

Sihhiye, ANKARA / TURKEY

Tel: (+90-312) 3052900

e-mail: phurmuz@yahoo.com,

pervin.hurmuz@hacettepe.edu.tr

\section{ORCIDs:}

Pervin Hurmuz

0000-0003-1221-9192

Caglayan Selenge Beduk Esen

Emre Unal

$0000-0001-9967-8177$

Musturay Karcaaltincaba

0000-0002-1520-2487

Gokhan Ozyigit

0000-0002-3384-0909

Fadıl Akyol

0000-0002-7497-4348

0000-0002-5287-3474 